\title{
Molecular, Solid-State and Surface Structures of the Conformational Polymorphic Forms of Ritonavir in Relation to their Physicochemical Properties
}

\author{
lan Rosbottom \\ Centre for the Digital Design of Drug Products, School of Chemical and Process Engineering, University of Leeds, Woodhouse Lane, \\ Leeds LS2 9JT, UK \\ irosbottom210289@gmail.com
}

\section{Purpose}

Molecular and crystallographic modelling can be used to de-risk the development of active pharmaceutical ingredients into drug products. Here we present an application of multi-scale modelling workflows to characterise polymorphism in ritonavir with regard to its stability, bioavailability and processing.

\section{Methods}

Molecular conformation, polarizability and stability are examined using quantum mechanics (QM). Intermolecular synthons, hydrogen bonding, crystal morphology and surface chemistry are modelled using empirical force fields.

\section{Results}

The form I conformation is more stable and polarized with more efficient intermolecular packing, lower void space and higher density, however its shielded hydroxyl is only a hydrogen bond donor. In contrast, the hydroxyl in the more open but less stable and polarized form II conformation is both a donor and acceptor resulting in stronger hydrogen bonding and a more stable crystal structure but one that is less dense. Both forms have strong 1D networks of hydrogen bonds and the differences in packing energies are partially offset in form II by its conformational deformation energy difference with respect to form I. The lattice energies converge at shorter distances for form I, consistent with its preferential crystallization at high supersaturation. Both forms exhibit a needle/lath-like crystal habit with slower growing hydrophobic side and faster growing hydrophilic capping habit faces with aspect ratios increasing from polar-protic, polar-aprotic and non-polar solvents, respectively. Surface energies are higher for form II than form I and increase with solvent polarity. The higher deformation, lattice and surface energies of form II are consistent with its lower solubility and hence bioavailability.

\section{Conclusion}

Inter-relationship between molecular, solid-state and surface structures of the polymorphic forms of ritonavir are quantified in relation to their physical-chemical properties.

Keywords: Pharmaceuticals, Crystallisation, Polymorphism, Ritonavir 